\title{
A Challenge to Us or a Challenge for Us? Negotiating Bias and Discrimination in the Consulting Room
}

\author{
Morgan T. Sammons \\ Published online: 7 May 2021 \\ (C) National Register of Health Service Psychologists 2021
}

The culture of scientific inquiry is in most respects inseparable from the prevailing social culture, but the culture of science generally evolves at a different and slower pace. Science tends to be governed by rubrics that are both more explicit and more static than unspoken societal mores. Social culture may be altered by scientific discoveries, but - to the extent that we can distinguish between them-scientific culture and social culture operate by different rules. There are always exceptions: Romantic science, as meticulously described in Richard Holmes' The Age of Wonder (Holmes, 2009), set much of the social stage throughout the Western industrial revolution in the $19^{\text {th }}$ century, but, in general, scientific culture lags behind that of the larger society. It appears that the events of the past year, however, have had a far more immediate effect on science. Replicability remains the cornerstone upon which we build knowledge. But the elimination of bias has taken on a far more universal meaning. And that is how it should be. In this issue of the Journal of Health Service Psychology, we address both structural and pragmatic changes that may better align the science of psychology with evolving societal norms.

David Drustrup (2021), a PhD candidate at the University of Iowa, presents us with a timely article on the challenges faced by white psychotherapists when dealing with racial issues in treatment. Drustrup communicates that it is critical to understand that the history of racial oppression in the United States (though it is a phenomenon clearly not limited to the United States) makes it extremely difficult for white therapists and white patients to understand the pervasiveness of bias that still exists in our culture. I suspect that there will be a variety of responses to this article and that some readers will have quite a strong reaction. Readers of color may have a response akin to "Why do I have to read about something I experience every day"; white readers may have responses more like "that's not me, I'm deeply aware of issues of bias and discrimination and have fully incorporated my awareness into my clinical work and interactions with non-white individuals." It is our obligation as therapists (and as human beings) to confront the racism around us, in our patients, and in ourselves.
Regardless of our ethnicity, we will all profit if we view Drustrup's article as a challenge for us rather than a challenge to us. Furthermore, therapists can apply Drustrup's central theme of racial discrimination to other forms of discrimination. Do we bring heteronormative biases regarding a patient's sexuality into the consulting room? Are biases regarding individual worth activated by our perceptions of a client's financial status?

If we presume that accurate understanding of therapists' implicit assumptions about minority groups is essential to accurate patient assessment, we must simultaneously address the requirement for accurate assessment of adverse childhood experiences (ACEs) in minority patients. As Toombs et al. (2021) argue, the dose-response relationship between ACEs and subsequent physical and emotional symptom development is particularly salient in Indigenous populations because they are historically the victims not only of economic and social oppression but also may express intergenerational health concerns that are based on a history of genocidal behavior, such as the deliberate introduction of the smallpox virus into Indigenous groups, an early and shameful example of biological warfare practiced in North America. Toombs and colleagues note that ACE assessments are statistically unlikely to be performed with Indigenous clientele, but argue that all assessments of Indigenous individuals should incorporate a trauma-informed approach in order to accurately conceptualize risk assessment and treatment plans.

Adverse childhood experiences are also at the heart of clinical exploration of complex posttraumatic stress disorder (CPTSD). In the case presented by Franco (2021), C-PTSD is hypothesized to result from a history of repeated childhood abuse. While the emotional sequelae of ACEs have been well explored in the literature, associations between physical disability and ACEs have begun to be systematically addressed more recently. Evidence of adverse effects of ACEs on neurobiological development, autoimmune disorders, and even mitochondrial DNA damage has been reported in the literature. Helping patients establish a link between chronic physical complaints and ACEs may assist them in contextualizing 
these disorders and enhance perceptions of self-efficacy in managing such disorders. As to the diagnosis of C-PTSD, we again enter into a disputed area. From its debut in the literature in 1980, PTSD has been the subject of multiple redefinitions, some based on science and others based on sociopolitical factors. While C-PTSD is now acknowledged in the ICD-11, further work is required to distinguish it from current definitions of PTSD.

The role of health psychology in the management of chronic physical disease is continually expanding. In this issue, Datta and Ogbeide (2021) outline a role for psychological services in the management of chronic kidney disease. Because a high percentage of patients undergoing dialysis present with psychological distress, and because nonadherence to medical regimens both complicates and hastens the course of chronic kidney disease, Datta and Ogbeide present strategies for incorporating recognized psychological protocols like motivational interviewing and change management into protocols designed to improve both outcome and quality of life in patients with renal disease.

In a like fashion, Riehl and Taft (2021) outline a specific role for an emerging subfield of health psychology: psychogastroenterology. Irritable bowel syndrome and inflammatory bowel disease are among the most common conditions involving a psychological referral, and the authors provide treatment strategies for both conditions. Assessment measures specific to GI disorders are discussed along with a précis of GI-specific cognitive behavioral psychotherapy. As in kidney disease, a number of extant protocols can be specifically adapted to address the needs of patients with common GI disorders.

In this issue of JHSP, we are pleased to announce the appointment of two associate editors: Dr. Kamini Krishnan and Dr. Melissa Santos. Kamini Krishnan, PhD, ABPP, is a boardcertified clinical neuropsychologist and Assistant Professor of Neurology at Cleveland Clinic Lerner College of Medicine. Melissa Santos, $\mathrm{PhD}$, is an Associate Professor of Pediatrics at the UCONN School of Medicine, the Division Chief of Pediatric Psychology, and Clinical Director of Obesity Services at Connecticut Children's. You may view their full biographies on the National Register's website (https://www. nationalregister.org/jhsp-info/editorial-team/). We are also announcing some structural changes to the journal. While we have never shied away from issues pertaining to diversity or underrepresented populations in health care, we commit that each issue of the journal from this point onward will feature at least one article, as the present issue does, addressing clinical issues surrounding disparities in health care service provision. We also have examined our own internal processes with an eye toward ensuring that all providers in psychology are adequately represented in our journal such that we claim a consistent voice in advancing the points of view of traditionally underrepresented populations in the field.

\section{References}

Datta. P. \& Ogbeide, S. A. (2021). Working Therapeutically With Patients With Kidney Disease. Journal of Health Service Psychology, 47(2). https://doi.org/10.1007/s42843-021-00036-3

Drustrup, D. (2021). Talking With white Clients About Race. Journal of Health Service Psychology, 47(2). https://doi.org/10.1007/s42843021-00037-2

Franco, F. (2021). Understanding and Treating C-PTSD. Journal of Health Service Psychology, 47(2). https://doi.org/10.1007/s42843021-00038-1

Holmes, R. (2009). The Age of Wonder: How the Romantic Generation Discovered the Beauty and Terror of Science. Pantheon.

Riehl, M.E. \& Taft, T.H. (2021). Working With Patients With Chronic Digestive Diseases. Journal of Health Service Psychology, 47(2). https://doi.org/10.1007/s42843-021-00034-5

Toombs, E., Lund, J., and Mushquash, C. J. (2021). Preliminary Recommendations for Assessing Adverse Childhood Experiences in Clinical Practice With Indigenous Clients. Journal of Health Service Psychology, 47(2). https://doi.org/10.1007/s42843-02100035-4

Publisher's Note Springer Nature remains neutral with regard to jurisdictional claims in published maps and institutional affiliations.

Morgan T. Sammons, PhD, ABPP, is the Chief Executive Officer of the National Register of Health Service Psychologists, and the Editor of the Journal of Health Service Psychology. He is a retired Navy captain and was formerly the U.S. Navy's specialty leader for clinical psychology. 